\title{
Clinical significance of P-glycoprotein and glutathione $S$-transferase $\pi$ expression in gallbladder carcinoma
}

\author{
QING-JIU MA ${ }^{1}$, YU-CUN ZHANG ${ }^{1}, \mathrm{JING}^{-S E N ~ S H I}{ }^{2}$ and GUO-CAI LI ${ }^{1}$ \\ ${ }^{1}$ General Surgery Department, GaoXin Hospital of Xi'an Jiao Tong University, Xi'an, Shaanxi 710075; \\ ${ }^{2}$ Hepatobiliary Department, First Hospital of Xi'an Jiao Tong University, Xi'an, Shaanxi 710061, P.R. China
}

Received August 1, 2013; Accepted December 10, 2013

DOI: $10.3892 /$ etm.2014.1472

\begin{abstract}
P-glycoprotein (P-gp) and glutathione $S$-transferase $\pi$ (GST- $\pi$ ) are not only drug-resistance markers, but also prognostic markers of various cancers. The aim of the present study was to investigate the clinical significance of P-gp and GST- $\pi$ in gallbladder carcinoma (GBC). Tissue samples from 42 patients with GBC were immunostained. Demographic, clinical and follow-up data were collected and analyzed. The positive expression rates of P-gp and GST- $\pi$ in the GBC tissues were significantly higher (76.2 and $64.3 \%$, respectively) than that of chronic cholecystitis specimens (30 and $20 \%$, respectively) $(\mathrm{P}=0.014$ and 0.035 , respectively), and correlated with the Nevin stage of GBC. Multivariate analysis demonstrated that patients with positive expression of $\mathrm{P}-\mathrm{gp}$ and GST- $\pi$ showed a significantly lower 2-year survival rate (11.1 and $12 \%$, respectively) compared with patients with negative expression (55.6 and 45.5\%, respectively) ( $\mathrm{P}=0.013$ and 0.036 , respectively). P-gp was also found to be an independent prognostic marker of 2-year survival rate by logistic regression analysis $(B=-2.76, P=0.061)$. Results of this study suggest that $\mathrm{P}-\mathrm{gp}$ is a prognostic marker of GBC and the detection of $\mathrm{P}-\mathrm{gp}$ and GST- $\pi$ may contribute to the prognosis of GBC and the application of chemotherapy as a therapeutic treatment.
\end{abstract}

\section{Introduction}

Gallbladder cancer (GBC) is a common and lethal cancer with a poor prognosis due to its insensitivity to radioactive and chemical therapies. The 5-year survival rate of GBC is $<5 \%(1,2)$. Early tumor resection is the only effective and potentially curative treatment. However, a number of patients present with GBC in the later stages when surgical intervention is no longer effective. Therefore, the identification of a

Correspondence to: Professor Guo-Cai Li, General Surgery Department, GaoXin Hospital of Xi'an Jiao Tong University, 16 South of Tuanjie Road, Xi'an, Shaanxi 710075, P.R. China E-mail: mrlgc@163.com

Key words: gallbladder cancer, P-glycoprotein, glutathione $S$-transferase, metastasis, prognosis biomarker with diagnostic and prognostic values is crucial for the treatment of GBC.

P-glycoprotein (P-gp) is encoded by the multidrug resistance 1 (MDR1) gene and is a membrane glycoprotein. It acts as an energy-dependent drug efflux pump and is important in pharmacokinetics. Overexpression of $\mathrm{P}$-gp is a major mechanism of drug resistance (3), thus, P-gp is used as a biomarker of drug resistance during the systemic treatment of various malignancies (4). In previous years, studies have identified that the expression of P-gp correlates with tumor progression and the prognosis of breast, colon and lung cancers (5-8)

Glutathione $S$-transferase $\pi$ (GST- $\pi$ ) is a subclass of GSTs, a polymorphic supergene family of detoxification enzymes involved in the metabolism of numerous potential carcinogens (9). GST- $\pi$ is mainly distributed in the placenta, lung, kidney, liver and red blood cells at a low levels of expression (10). In addition, GST- $\pi$ is the most important phase II drug-metabolizing enzyme and is involved in the metabolism and detoxification of environmental carcinogens and chemotherapeutics. Previously, it was reported that the expression levels of GST- $\pi$ correlate with the prognosis of malignances, including glioblastoma and breast, prostate and colorectal cancer (9,11-13).

However, the clinical significance of P-gp and GST- $\pi$ in the progression and prognosis of GBC remain unknown. Therefore, the present study aimed to investigate the expression levels and prognostic values of P-gp and GST- $\pi$ in GBC.

\section{Materials and methods}

Samples. In total, 42 tissue samples of GBC were obtained from patients at the First Hospital of Xi'an Jiao Tong University (Xi'an, China) between January 2000 and July 2005. Following excision, samples were paraffin-embedded. The diagnosis of GBC was established by histopathological analysis and surgery was performed according to the stage defined by the Nevin classification (14). In addition, 10 tissue samples obtained from patients with chronic cholecystitis were used as the controls.

Demographic and clinical data, including age, gender, the presence of gallstones and disease history, were obtained. The patient cohort included 26 females and 16 males and the mean age at surgical resection was $60.81 \pm 1.30$ years. All patients did not present with complications, such as hypertension, diabetes and chronicle hepatitis. Histological types of GBC were clas- 
sified according to previous studies $(15,16)$, which included adenocarcinoma (not otherwise specified), papillary adenocarcinoma, adenosquamous, mucinous, adenocarcinomas and undifferentiated carcinoma.

This study was approved by the Ethical Committee of Xi'an JiaoTong University. All patients received oral and written information regarding the study protocol and signed an informed consent prior to inclusion in the study.

Immunohistochemistry. Paraffin-embedded tumor tissues were sliced into 5- $\mu \mathrm{m}$ thick sections and mounted on glass. Slides were deparaffinized and rehydrated in $10 \mathrm{~min}$ through a graded alcohol series to deionized water in $1 \%$ Antigen Unmasking Solution (Vector Laboratories, Burlingame, CA, USA) and microwaved to enhance antigen retrieval. Tissue samples were sequentially incubated with anti-mouse immunoglobulin coupled to horseradish peroxidase (HRP). Slides were incubated with the specific primary anti-GST- $\pi$ (ab47709; Abcam, Cambridge, UK) and anti-P-gp (P7965; Sigma, St. Louis, MO, USA) monoclonal antibodies with an HRP-conjugated secondary antibody (A1293; Sigma), and then stained with 3,3-diaminobenzidine and counterstained with hematoxylin and eosin. In addition, 10 tissue samples from patients with chronic cholecystitis obtained by cholecystectomy were used as the control group. Two pathologists independently observed and interpreted the results of the immunohistochemical staining.

Assessment of staining. Staining of P-gp and GST- $\pi$ was evaluated according to the percentage of positive cells under an optical microscope (Leica Microsystems, GmbH, Wetzlar, Germany; magnification, x20). Staining intensity was classified as the following: Negative (-), no immunopositive staining or $<10 \%$ of positive cells observed; weak to moderate $(+)$, $10-30 \%$ positive cells; and high $(++),>30 \%$ positive cells.

Patient follow-up. Patients were advised to undergo 2-year regular follow-ups following GBC diagnosis. Follow-up data from 36 patients were obtained and the remaining data were lost.

Statistical analysis. Statistical analysis was performed using SPSS software (version 11.5; SPSS, Inc., Chicago, IL, USA). Differences of expression rate among groups were analyzed by Pearson's $\chi^{2}$ test. The Fisher's exact test was used to assess the differences between the positive rates when the number of total cases was $<40$. All statistical tests were two-sided. To elucidate the risk factors for prognosis (2-year survival rate), multivariate analysis was performed using the logistic regression model. $\mathrm{P}<0.05$ was considered to indicate a statistically significant difference.

\section{Results}

Expression levels of $P$-gp and GST- $\pi$ in GBC. P-gp and GST- $\pi$ were mainly expressed in the cytoplasm or membrane of GBC cells (Fig. 1). The positive expression rate of P-gp and GST- $\pi$ in the GBC tissues were 76.2 and $64.3 \%$, respectively, which was significantly higher than that in the chronic cholecystitis tissues ( 30 and $20 \%$, respectively) $(\mathrm{P}=0.014$ and $\mathrm{P}=0.035$, respectively) (Fig. 2A). The expression levels were not correlated with gender, age, pathology, presence of gallstones and histological grading (Table I).

Expression levels of P-gp and GST- $\pi$ correlate with the Nevin stage. The expression levels of P-gp and GST- $\pi$ in the early Nevin stages of GBC (I, II and III) were lower (33.3 and $16.7 \%$, respectively) than that in the later stages (IV and V) (83.3 and $72 \%$, respectively) ( $\mathrm{P}=0.021$ and 0.016 , respectively) (Fig. 2B). As Nevin staging is classified by tumor metastasis, multivariate analysis was performed using the logistic regression model and found that $\mathrm{P}$-gp staining is an independent risk factor for metastasis of GBC $\left(\mathrm{R}^{2}=3.09 ; \mathrm{P}=0.044\right)$ (Table II).

$P$-gp and GST- $\pi$ expression positively correlates with the prognosis of GBC. According to follow-up data of 36 cases, the expression levels of P-gp and GST- $\pi$ significantly correlated with the 2-year survival rate. The 2-year survival rate in P-gp-negative patients (55.6\%) was higher than that in the P-gp-positive patients (11.1\%) $(\mathrm{P}=0.013)$. Similarly, 2-year survival rate in the GST- $\pi$-negative patients $(45.5 \%)$ was also higher than that in the GST- $\pi$-positive patients (12.0\%) $(\mathrm{P}=0.036)$. In addition, coexpression of P-gp and GST- $\pi$ demonstrated the lowest 2-year survival rate $(4.3 \%)(\mathrm{P}=0.001)$ (Fig. 2C). P-gp was also found to be an independent prognostic marker of the 2-year survival rate by logistic regression analysis $\left(\mathrm{R}^{2}=-2.76, \mathrm{P}=0.061\right)$ (Table III).

Correlation between P-gp and GST- $\pi$. A significant positive correlation was also found between the expression levels of P-gp and GST- $\pi$ in tumor tissues $\left(\mathrm{R}^{2}=0.20 ; \mathrm{P}=0.003\right)$ and the coexpression rate was $59.5 \%$ (Fig. 2D).

\section{Discussion}

In the present study, expression levels of P-gp and GST- $\pi$ in malignant lesions were found to be higher than that of benign lesions, indicating multiple drug resistance of GBC. In addition, majority of positive cells were located on the mucosal surface of the gallbladder, this phenomena was consistent with the role of P-gp as a multidrug transporter and support the mechanism of GBC. Similar results have also previously been identified suggesting that P-gp is substantially expressed on the biliary surface of hepatocytes and small biliary ductules (17).

Although P-gp and GST- $\pi$ correlate with drug resistance, their mechanisms and drug-resistant spectrum are different. GST- $\pi$ is regulated in vivo by reactive oxygen species and its induction represents part of an adaptive response mechanism to chemical stress caused by electrophiles (18). The drug-resistance spectrum of GST- $\pi$ is cisplatin. However, the drug-resistance spectrum of P-gp is vincristine and doxorubicin $(19,20)$. Therefore, the expression of P-gp and GST- $\pi$ should be taken into consideration when designing clinical trials.

It has been previously suggested that aromatic compounds can induce GST- $\pi$ expression $(21,22)$. In addition, it is well known that GBC is closely associated with gallstone and chronic cholecystitis, which may generate aromatic compounds due to the long-term stasis of bile and bacterial infection (23). Thus, we hypothesized that aromatic compounds are not only 
Table I. Demographic and clinical data and expression of P-gp and GST- $\pi$ of gallbladder carcinoma patients.

\begin{tabular}{|c|c|c|c|c|c|c|c|c|c|c|c|}
\hline \multirow[b]{2}{*}{ Parameter } & \multirow{2}{*}{$\begin{array}{c}\text { Cases, } \\
\mathrm{n}\end{array}$} & \multicolumn{3}{|c|}{ P-gp staining } & \multirow{2}{*}{$\begin{array}{l}\text { Positive rate, } \\
\%\end{array}$} & \multirow[b]{2}{*}{ P-value } & \multicolumn{3}{|c|}{ GST- $\pi$ staining } & \multirow{2}{*}{$\begin{array}{c}\text { Positive rate } \\
\%\end{array}$} & \multirow[b]{2}{*}{ P-value } \\
\hline & & - & + & ++ & & & - & + & ++ & & \\
\hline \multicolumn{12}{|l|}{ Gender } \\
\hline Male & 16 & 3 & 11 & 2 & 81 & - & 6 & 8 & 2 & 63 & - \\
\hline Female & 26 & 7 & 11 & 8 & 73 & 0.22 & 9 & 12 & 5 & 65 & 0.86 \\
\hline \multicolumn{12}{|l|}{ Age, years } \\
\hline$\geq 60$ & 26 & 5 & 12 & 9 & 81 & - & 7 & 16 & 3 & 73 & - \\
\hline$<60$ & 16 & 5 & 10 & 1 & 69 & 0.11 & 8 & 4 & 4 & 50 & 0.10 \\
\hline \multicolumn{12}{|l|}{ Presence of gallstones } \\
\hline Yes & 20 & 5 & 8 & 7 & 75 & - & 7 & 9 & 4 & 65 & - \\
\hline No & 22 & 5 & 14 & 3 & 78 & 0.21 & 8 & 11 & 3 & 64 & 0.85 \\
\hline \multicolumn{12}{|l|}{ Pathology } \\
\hline Adenocarcinoma (NOS) & 28 & 5 & 17 & 6 & 82 & - & 9 & 14 & 5 & 68 & - \\
\hline Papillary & & & & & & & & & & & \\
\hline adenocarcinoma & 5 & 2 & 2 & 1 & 60 & - & 3 & 2 & - & 40 & - \\
\hline Adenosquamous & 3 & 1 & 2 & - & 67 & - & 2 & 1 & - & 33 & - \\
\hline $\begin{array}{l}\text { Mucinous } \\
\text { adenocarcinoma }\end{array}$ & 2 & - & 1 & 1 & 100 & - & - & 1 & 1 & 100 & - \\
\hline $\begin{array}{l}\text { Undifferentiated } \\
\text { carcinoma }\end{array}$ & 4 & 2 & - & 2 & 50 & 0.48 & 1 & 2 & 1 & 75 & 0.41 \\
\hline \multicolumn{12}{|l|}{ Histological grade } \\
\hline I & 9 & 4 & 4 & 1 & 56 & - & 4 & 5 & - & 56 & - \\
\hline II & 19 & 3 & 12 & 4 & 84 & - & 6 & 10 & 3 & 68 & - \\
\hline III & 14 & 3 & 6 & 5 & 79 & 0.35 & 5 & 5 & 4 & 64 & 0.46 \\
\hline
\end{tabular}

P-gp, P-glycoprotein; GST- $\pi$, glutathione $S$-transferase $\pi$; NOS, not otherwise specified.

Table II. Logistic regression analysis of metastasis of gallbladder carcinoma.

\begin{tabular}{lccc}
\hline Variable & Regression coefficent & Standard error & P-value \\
\hline P-gp & 3.09 & 1.53 & 0.044 \\
Tumor grade & 0.48 & 1.00 & 0.631 \\
Age & 0.03 & 0.08 & 0.705 \\
Gender & 2.61 & 1.69 & 0.123 \\
Pathological type & 0.55 & 1.59 & 0.727 \\
Gallstones & 2.41 & 1.71 & 0.158 \\
\hline
\end{tabular}

P-gp expression was coded as: 1, postive; 2 , negative. Tumor grade was coded 1-3, with increasing grade. Gender was coded as: 1, male; 2 , female. Pathological type was coded as: 1 , adnocarcinoma; 2 , non-adnocarcinoma. Presence of gallstones was coded as: 0 , no; 1 , yes. P-gp, P-glycoprotein .

comprised of chemical factors for the carcinogenesis of $\mathrm{GBC}$, but are also important for GST- $\pi$ expression.

The main prognostic factors of GBC are the clinical or pathological stages (24) and the Nevin staging system for GBC is widely used (25). The Nevin stage of GBC is mainly defined according to metastasis and invasion. In the present study, P-gp and GST- $\pi$ showed lower levels in non-metastatic tumors (Nevin stage, I, II and III) than in metastatic tumors (Nevin stage, IV and V), suggesting that P-gp and GST- $\pi$ may be used as indicators for invasion and metastasis. In the present study, patients with positive P-gp or GST- $\pi$ expression showed a shorter 2-year survival rate compared with patients with a negative expression. Furthermore, patients with coexpression of P-gp and GST- $\pi$ were associated with the worst prognosis. These results demonstrate that P-gp and GST- $\pi$ may be used as prognostic markers for GBC and were consistent with previous studies on liver, colon, breast and ovarian cancer (26-28).

A close correlation between GST- $\pi$ and P-gp expression was also identified in the current study, with coexpression observed in $59.5 \%$ of patients with GBC. Similar studies have 
Table III. Logistic regression analysis of 2-year survival of gallbladder carcinoma patients.

\begin{tabular}{lccr}
\hline Variable & Regression coefficent & Standard error & P value \\
\hline P-gp & -2.76 & 1.47 & 0.061 \\
Tumor stage & 0.84 & 2.13 & 0.693 \\
Tumor grade & -1.28 & 0.90 & 0.153 \\
Age & 0.01 & 0.06 & 0.959 \\
Gender & -0.46 & 1.15 & 0.687 \\
Pathological type & 0.16 & 1.19 & 0.896 \\
Gallstones & 0.05 & 1.18 & 0.969 \\
\hline
\end{tabular}

P-gp expression was coded as: 1 , postive; 2 , negative. Tumor stage was coded as: 1 , I+II+III; 2, IV+V; according to Nevin staging. Tumor grade was coded, 1-3 with increasing grade. Gender was coded as: 1, male; 2, female. Pathological type was coded as: 1, adnocarcinoma; 2 , non-adnocarcinoma. Presence of gallstones was coded as: 0, no; 1, yes. P-gp, P-glycoprotein.
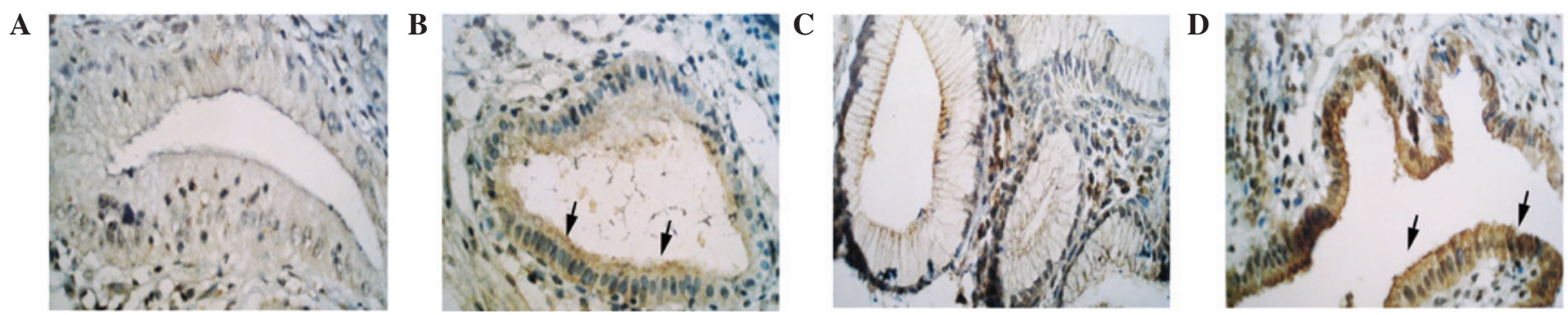

Figure 1. Immunohistochemical analysis of expression of P-gp and GST- $\pi$ in GBC tissues. (A) Negative expression of P-gp; (B) Positive expression of P-gp was observed in gallbladder carcinoma. The majority of positive cells were located on the surface of the mucosa; C) Negative expression of GST- $\pi$ in gallbladder carcinoma; (D) Positive expression of GST- $\pi$ in gallbladder carcinoma (magnification, x20). Arrows denote positive cells. P-gp, P-glycoprotein; GST- $\pi$, glutathione $S$-transferase $\pi$; GBC, gallbladder carcinoma.

A

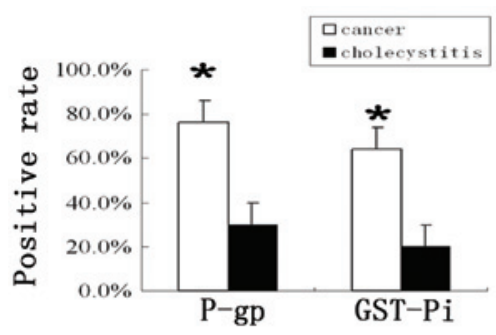

C

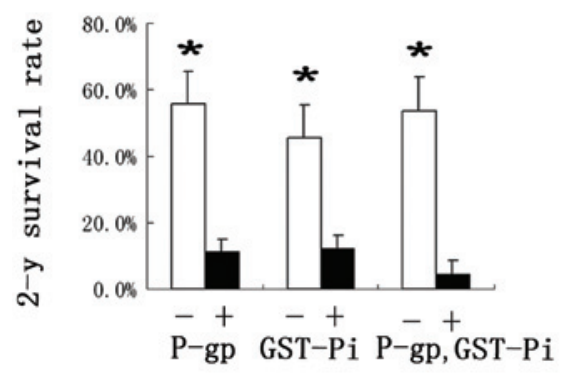

B

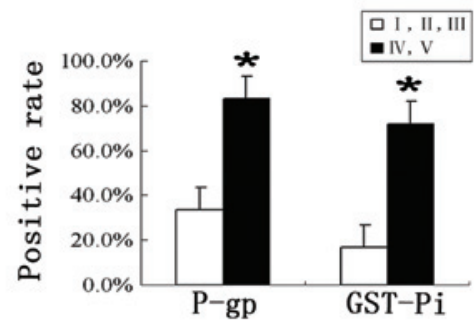

D

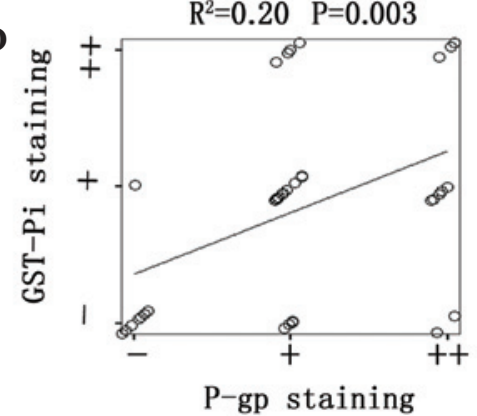

Figure 2. Expression levels of P-gp and GST- $\pi$ correlate with metastasis and prognosis. (A) Comparison of P-gp and GST- $\pi$ expression levels between GBC and cholecystitis tissues ("P<0.05, vs. cholecystitis). (B) Comparison of P-gp and GST- $\pi$ expression levels between GBC and the early Nevin stage (I,II and III) and late Nevin stage (IV and V). ${ }^{*} \mathrm{P}<0.05$ compared with early Nevin stage (I, II and III). (C) Differences in the 2-year survival rate between GBC tissues with a positive or negative expression of P-gp and GST- $\pi$. $* \mathrm{P}<0.05$ compared with positive specimen. (D) Correlation between the expression of P-gp and GST- $\pi$. Dots denote each sample and the line represents the regression line. P-gp, P-glycoprotein; GST- $\pi$, glutathione $S$-transferase $\pi$; GBC, gallbladder carcinoma; R: correlation coefficient.

shown that the rate of coexpression was $93 \%$ in patients with leukemia and $80 \%$ in patients with lung cancer $(29,30)$. It has been demonstrated there are abnormal expression of genes, such as c-erbB-2, neu, P53, ras, INT2, HSTF1, bcl-2, c-fos and c-jun 
in various types of cancer, including GBC, and these genes not only correlate with drug resistance but also frequently regulate and co-amplify P-gp and GST- $\pi$ genes (28-34). Therefore, the higher expression levels of P-gp and GST- $\pi$ in patients with GBC may be a reflection of the abnormal expression of oncogenes and cancer suppressor genes.

In conclusion, results of the present study suggest that $\mathrm{P}$-gp is a prognostic marker for GBC. In the future, the detection of P-gp and GST- $\pi$ in patients with GBC may contribute to chemotherapeutic and surgical decisions.

\section{Acknowledgements}

The authors thank Mei-Rong Han, He-Ping Tian, Yi-Jun Yang and Yue Han for their assistance in the immunostaining experiments and clinical data collection.

\section{References}

1. Mekeel KL and Hemming AW: Surgical management of gallbladder carcinoma: a review. J Gastrointest Surg 11: 1188-1193, 2007.

2. Miller G and Jarnagin WR: Gallbladder carcinoma. Eur J Surg Oncol 34: 306-312, 2008

3. Khdair A, Handa H, Mao G and Panyam J: Nanoparticle-mediated combination chemotherapy and photodynamic therapy overcomes tumor drug resistance in vitro. Eur J Pharm Biopharm 71: 214-222, 2008

4. Schrader AJ, Seger M, Konrad L, et al: Clinical impact of MDR1-expression in testicular germ cell cancer. Exp Oncol 29: 212-216, 2007.

5. Bark H, Xu HD, Kim SH, Yun J and Choi CH: P-glycoprotein down-regulates expression of breast cancer resistance protein in a drug-free state. FEBS Lett 582: 2595-2600, 2008.

6. Berger W, Setinek U, Hollaus P, et al: Multidrug resistance markers P-glycoprotein, multidrug resistance protein 1, and lung resistance protein in non-small cell lung cancer: prognostic implications. J Cancer Res Clin Oncol 131: 355-363, 2005.

7. Bottke D, Koychev D, Busse A, et al: Fractionated irradiation can induce functionally relevant multidrug resistance gene and protein expression in human tumor cell lines. Radiat Res 170: 41-48, 2008.

8. Hanson JA, Gillespie JW, Grover A, et al: Gene promoter methylation in prostate tumor-associated stromal cells. J Natl Cancer Inst 98: 255-261, 2006.

9. Pandey SN, Jain M, Nigam P, Choudhuri G and Mittal B: Genetic polymorphisms in GSTM1, GSTT1, GSTP1, GSTM3 and the susceptibility to gallbladder cancer in North India. Biomarkers 11: 250-261, 2006.

10. Tiirikainen MI, Elonen E, Syrjälä MT, Jansson SE and Krusius T: Flow cytometric analysis of glutathione-S-transferase-pi in acute leukemia. Leukemia 8: 978-984, 1994.

11. Somlo G, Chu P, Frankel P, et al: Molecular profiling including epidermal growth factor receptor and p21 expression in high-risk breast cancer patients as indicators of outcome. Ann Oncol 19 1853-1859, 2008.

12. Perry AS, Loftus B, Moroose R, et al: In silico mining identifies IGFBP3 as a novel target of methylation in prostate cancer. Br J Cancer 96: 1587-1594, 2007.

13. Sutoh I, Kohno H, Nakashima Y, et al: Concurrent expressions of metallothionein, glutathione S-transferase-pi, and P-glycoprotein in colorectal cancers. Dis Colon Rectum 43: 221-232, 2000.
14. Nevin JE, Moran TJ, Kay S and King R: Carcinoma of the gallbladder: staging, treatment, and prognosis. Cancer 37: 141-148, 1976.

15. Henson DE, Albores-Saavedra J and Corle D: Carcinoma of the gallbladder. Histologic types, stage of disease, grade, and survival rates. Cancer 70: 1493-1497, 1992.

16. Levy AD, Murakata LA and Rohrmann CA Jr: Gallbladder Carcinoma: radiologic-pathologic correlation. Radiographics 21: 295-314, 2001.

17. Gottesman MM and Pastan I: The multidrug transporter, a double-edged sword. J Biol Chem 263: 12163-12166, 1988.

18. Rodriguez C, Commes T, Robert J and Rossi JF: Expression of P-glycoprotein and anionic glutathione S-transferase genes in non-Hodgkin's lymphoma. Leuk Res 17: 149-154, 1993.

19. Kufe DW, Pollock RE, Weichselbaum RR, et al (eds): Holland-Frei Cancer Medicine. 6th edition. Hamilton (ON): BC Decker, pp711-726, 2003.

20. Stewart DJ: Mechanisms of resistance to cisplatin and carboplatin. Crit Rev Oncol Hematol 63: 12-31, 2007.

21. Chen YK and Lin LM: Placental glutathione S-transferase isoenzyme expression in polycyclic aromatic hydrocarbon-induced hemster buccal pouch mucosa. Oral Oncol 34: 180-185, 1998.

22. Tsuchida S and Sato K: Glutathione transferses and cancer. Crit Rev Biochem Mol Biol 27: 337-384, 1992.

23. Takabayashi A, Watkins JB, Soloway RD, Rios-Dalenz J and Henson DE: Glycolithocolic acid is greatly increased in stones from patients with carcinoma of the gallbladder. Gastroenterology 79: 1058-1063, 1980

24. Henson DE, Albores-Saavedra J and Corle D: Carcinoma of the gallbladder. Histologic types, stage of disease, grade, and survival rates. Cancer 70: 1493-1497, 1992.

25. Lazcano-Ponce EC, Miquel JF, Muñoz N, et al: Epidemiology and molecular pathology of gallbladder cancer. CA Cancer J Clin 51: 349-364, 2001.

26. Seo S, Hatano E, Higashi T, et al: Fluorine-18 fluorodeoxyglucose positron emission tomography predicts tumor differentiation, P-glycoprotein expression, and outcome after resection in hepatocellular carcinoma. Clin Cancer Res 13: 427-433, 2007.

27. Sinicrope FA, Hart J, Brasitus TA, Michelassi F, Lee JJ and Safa AR: Relationship of P-glycoprotein and carcinoenbryonic antigen expression in human colon carcinoma to local invision, DNA ploidy, and disease relapse. Cancer 74: 2908-2907, 1994.

28. Saint-Ruf C, Malfoy B, Scholl S, Zafrani B and Dutrillaux B: GST gene is frequently complified with INT2 and HSTF1 proto-oncogene in human breast cancers. Oncogene 16: 403-406, 1991.

29. Slebos RJ, Kibbelaar RE, Dalesio O, et al: K-ras oncogene activation as a prognostic marker in adenocarcinomar of the lung. $\mathrm{N}$ Eng J Med 323: 561-565, 1990.

30. Tsuda H, Hirohashi S, Shimosato Y, et al: Correlation between long-term survival in breast cancer patients and amplification of two putive oncogene-coamplification units: hst-1/int-2 and c-erbB-2/ear-1. Cancer Res 49: 3104-3108, 1989.

31. Charpin C, Vielh P, Duffaud F, et al: Quantitative immunocytochemical assays of P-glycoprotein in breast carcinoma: correlation to messanger RNA experession and to immunohistochemical prognostic indicators. J Natl Cancer Inst 86: 1539-1545, 1994.

32. Ralhan R, Swain RK, Agarwal S, et al: P-glycoprotein is positively correlated with p53 in human oral pre-malignant and malignant lesions and is associated with poor prognosis. Int J Cancer 84: 80-85, 1999.

33. Malik IA: Gallbladder cancer: current status. Expert Opin Pharmacother 5: 1271-1277, 2004.

34. Brotherick I, Shenton BK, Egan M, et al: Examination of multidrug resistance in cell lines and primary breast tumours by flow cytometry. Eur J Cancer 32A: 2334-2341, 1996. 